\title{
Generation of Single Picosecond and Subpicosecond Light Pulses
}

\author{
A. Penzkofer, D. von der Linde, A. Laubereau, and W. Kaiser \\ Physik-Department der Technischen Universität München, Germany \\ (Received 31 January 1972)
}

\begin{abstract}
Picosecond light pulses passing through a saturable absorber show considerable pulse shortening. For instance, under special favorable conditions the pulse duration was found to be reduced from 8 to $2.6 \mathrm{psec}$ in a single transit. Using a multiple-absorber-amplifier system, light pulses were shortened from 8 to 0.7 psec in five transits. The shortest pulse seen in our experiments had a duration of less than $0.5 \mathrm{psec}$. Calculations based on a twolevel approximation of the absorbing medium agreed well with the experimental results.
\end{abstract}

The common technique for generating pulses of psec duration consists of mode locking a Nd : glass laser. ${ }^{1}$ Sérious difficulties were encountered when pulses of a duration of 1 psec or less were attempted by this method. It was found that the pulse duration changes drastically during the course of the pulse train ${ }^{2}$ : At the beginning of the train, pulses of several psec duration $(\sim 5 \mathrm{psec})$ are generally observed, while at the end of the train the pulses disintegrate with subpicosecond substructure. ${ }^{3,4}$ Two techniques have been reported to generate pulses of subpicosecond duration: (i) the optical compression of frequency-modulated pulses. ${ }^{5}$ On account of the difficulty of accurately adjusting the compression to the chirp, the shape and peak power of the generated pulses are quite uncertain: (ii) the pulse shortening through the transient stimulated Raman effect. ${ }^{6}$ This system works with the whole train of pulses; it has to await further investigations.

In this letter we wish to present theoretical and experimental investigations of the shortening of single psec light pulses using saturable absorbers of low transmission in conjunction with standard laser amplifiers. First, the pulse shortening in a single pass through a saturable absorber is investigated. Then, the reduction in pulse duration is studied for several passes through absorbers and amplifiers.

In our calculations the saturable absorber ${ }^{7}$ is described by a two-level system, and the transient transmission is determined following Refs. 8 and 9 . Recently, it has been shown that this model accounts quantitatively for a measuring system of peak intensities of psec light pulses. ${ }^{9}$ The intensity transmission $T$ of the saturable absorber depends on several factors: the initial transmission $T_{0}$ of the dye at low-intensity level, the peak intensity $I_{0}$ of the incident pulse, the shape of the input pulse $s\left(t^{\prime}, r^{\prime}\right)=s\left(t / \Delta t_{\mathrm{in}}, r / \Delta r_{\mathrm{in}}\right)$, where $\Delta t_{\mathrm{in}}$ and $\Delta r_{1 \mathrm{n}}$ are the pulse duration (FWHM) and the beam radius
(HWHM) of the input pulse, respectively, and the ratio $\tau / \Delta t_{\text {in }}$ of the lifetime $\tau$ of the excited state of the dye to the pulse duration $\Delta t_{1 \mathrm{n}}$. We have studied the intensity transmission $T$ numerically for a wide range of parameters. In this letter we are interested in the transmitted intensity $I_{\mathrm{tr}}$ :

$$
I_{\mathrm{tr}}=T\left[T_{0}, I_{0} s\left(t^{\prime}, r^{\prime}\right), \tau / \Delta t_{\mathrm{in}}\right] I_{0} s\left(t^{\prime}, r^{\prime}\right) \text {. }
$$

In Fig. 1 we present calculations of the normalized transmitted intensity $I_{\mathrm{tr}} / I_{0}$ as a function of the normalized time $t / \Delta t_{\text {in }}$ for several values of the input peak intensity $I_{0}$; the parameters $T_{0}, \tau / \Delta t_{\mathrm{in}}$, and $s\left(t^{\prime}, r^{\prime}\right)$ were kept constant: $T_{0}=10^{-7}, \tau=9.1$ psec (see Ref. 7), $\Delta t_{\mathrm{in}}=8 \mathrm{psec}$ and Gaussian input pulse shape. For ready comparison the incident Gaussian pulse is included in Fig. 1 (broken line). It is clearly seen from Fig. 1 that for high input intensities (e.g., $5 \times 10^{9} \mathrm{~W} / \mathrm{cm}^{2}$ ) the peak intensity and pulse duration are little affected by the saturable absorber; only the leading part of the pulse experiences the strong absorption of the dye. On the other hand, at lower input intensity (e.g., $1.2 \times 10^{9}$ $\mathrm{W} / \mathrm{cm}^{2}$ ) the transmitted pulse is drastically reduced in intensity (factor of 300 ) with smaller reduction in pulse duration (see, also, Fig. 2). There is a definite optimum for the shortening of the input pulse: At $I_{0}=1.8$ $\times 10^{9} \mathrm{~W} / \mathrm{cm}^{2}$ the transmitted pulse is reduced to a duration of $\Delta t_{\mathrm{tr}}=0.35 \Delta t_{\mathrm{in}}$ (see Fig. 1). Under these conditions the output peak intensity is down by a factor of 5 , and the total energy is reduced by a factor of 400 for a Gaussian cross section of the input beam.

In Fig. 2, the pulse-shortening ratio $\Delta t_{\mathrm{tr}} / \Delta t_{\text {in }}$ is depicted as a function of the peak intensity of the input pulse $I_{0}$ for various initial transmissions $T_{0}$. The curves are calculated for an input pulse duration of $\Delta t_{\mathrm{in}}=8 \mathrm{psec}$, for a Gaussian input pulse shape, and for parameters of the saturable absorber given in Ref. 7. Figure 2 shows quite convincingly that the pulse shortening obtainable in 


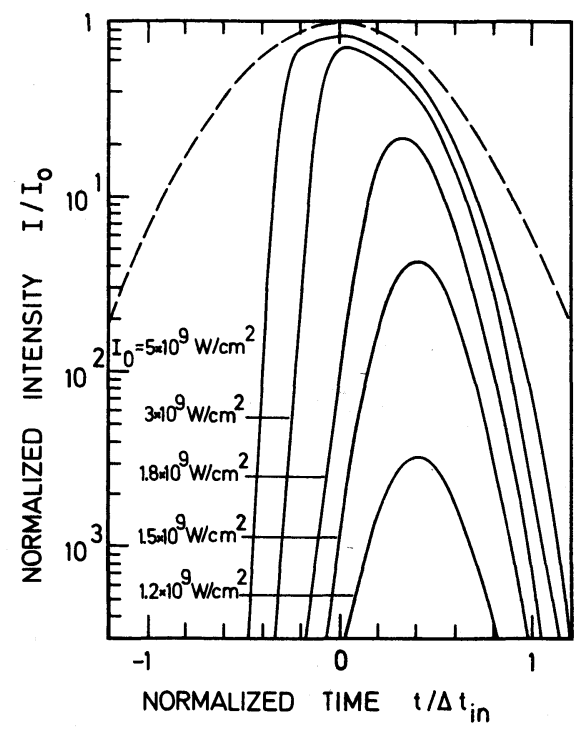

FIG. 1. Changes of the pulse shape after a single pass through a saturable absorber. Broken curve: normalized intensity of the input pulse $\left(\Delta t_{\text {in }}=8 \mathrm{psec}\right.$, Gaussian pulse shape). Solid curves: normalized intensity of the transmitted pulse for several values of the input peak intensity $I_{0}$. Dye parameters: $T_{0}=10^{-7}, \sigma=1.84 \times 10^{-16} \mathrm{~cm}^{2}, \tau=9.1$ psec.

a single pass depends critically on the incident peak intensity $I_{0}$ and on the initial dye transmission $T_{0}$. The minima of the various curves indicate optimum input peak intensities $I_{0}=I_{0}$ (opt) for given transmissions $T_{0}$. These minima are rather broad at larger transmission values with smaller pulse reductions. For small values of $T_{0}$ the sharp minima require a very careful control of the input peak intensity $I_{0}$; if $I_{0}<I_{0}$ (opt) the whole pulse is strongly attenuated, if $I_{0}>I_{0}$ (opt) the effect of pulse shortening decreases rapidly, and transmitted pulses strongly asymmetric in time are generated (see Fig. 1). It should be emphasized that at $I_{0}$ (opt) the peak intensity $I_{0, t r}$ and the energy of the transmitted pulse are only moderately reduced, e.g., for $T_{0}=10^{-1}$ or $10^{-40}$ we calculate $I_{0, \operatorname{tr}}=0.6 I_{0}$ or $0.1 I_{0}$ and an energy loss by a factor of 3 or $4 \times 10^{3}$, respectively (Gaussian beam profile).

We have made a series of experiments with saturable absorbers of initial transmissions $T_{0}=10^{-3}$ (open circle in Fig. 2) and $T_{0}=10^{-7}$ (full circles in Fig. 2). The pulse durations of the incident pulse $\Delta t_{\text {in }}$ and the transmitted pulse $\Delta t_{\mathrm{tr}}$ were determined by the two-photon fluorescence technique. ${ }^{10}$ The peak intensity of the incident pulse was measured by the method of energy transmission through saturable absorbers. ${ }^{9}$ Figure 2 shows very good agreement between the experimental points and the calculated curves. The predicted intensity dependence of the pulse shortening is confirmed experimentally (see curve with $T_{0}=10^{-7}$ ). The optimum pulse shortening in a single pass was found to be close to a factor of $3\left(T_{0}=10^{-7}\right)$.

We have extended our calculations to consider the effect of different shapes and durations of the input pulses. Our results are briefly summarized as follows: (1) The pulse-shortening ratio $\Delta t_{\mathrm{tr}} / \Delta t_{\mathrm{in}}$ comes out to be similar

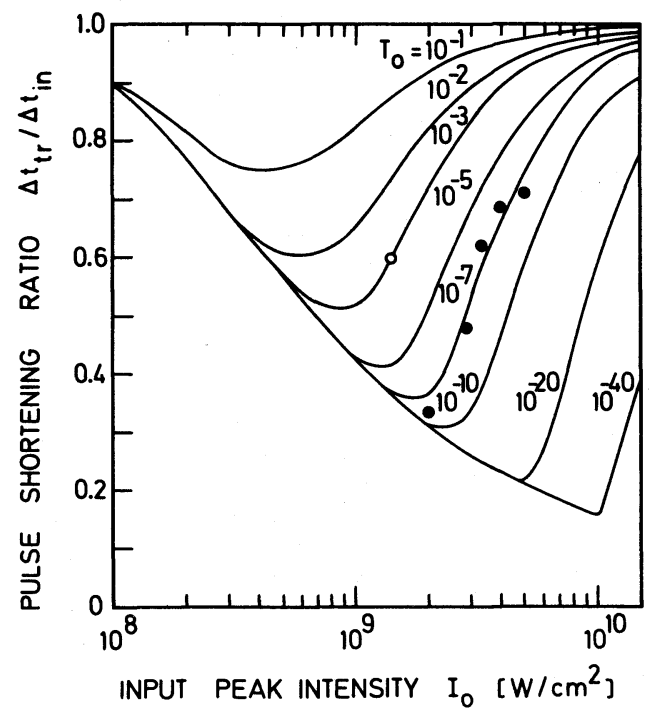

FIG. 2. Pulse-shortening ratio $\Delta t_{\mathrm{tr}} / \Delta t_{\mathrm{in}}$ as a function of the input peak intensity $I_{0}$ for several values of initial dye transmission $T_{0}$ (single pass). Input pulse: $\Delta t_{\text {in }}=8 \mathrm{psec}$, Gaussian shape. Experimental points are indicated for $T_{0}=10^{-3}$ and $T_{0}=10^{-7}$ by open and closed circles, respectively.

for Gaussian, Lorentzian, and hyperbolic secant pulses over a wide range of values for $\Delta t_{\mathrm{in}} / \tau$ and $T_{0}$ (from steady state to $\Delta t_{\mathrm{in}} / \tau=0.1$, and from $T_{0}=1$ to $T_{0}$ $=10^{-10}$ ). (2) The pulse-shortening ratio increases slightly for shorter input pulses (from $\Delta t_{\mathrm{tr}} / \Delta t_{\mathrm{in}} \simeq 0.3$ at the steady state to $\Delta t_{\mathrm{tr}} / \Delta t_{\mathrm{in}} \simeq 0.45$ at $\Delta t_{\mathrm{in}} / \tau=0.1$ for $T_{0}=10^{-7}$ ). (3) For decreasing durations of the input pulses, higher values for $I_{0}$ (opt) are required. (4) Shorter input pulses give more favorable ratios of $I_{0, \mathrm{tr}} / I_{0}$, i.e., higher output intensities at $I_{0}$ (opt). While in the steady-state case $\left(\Delta t_{\mathrm{in}} \gg \tau\right)$, the pulse shortening occurs only on account of the intensity dependence of



FIG. 3. Pulses generated in five transits through an absorberamplifier system. Broken curve: input pulse of 8-psec duration and Gaussian shape. Solid curves: pulses after $1-5$ passes through absorbers (and 0-4 transits through amplifiers). Dye transmission per pass: $T_{0}=2 \times 10^{-4}$. Amplifier gain per pass: $\gamma=4.1$ 


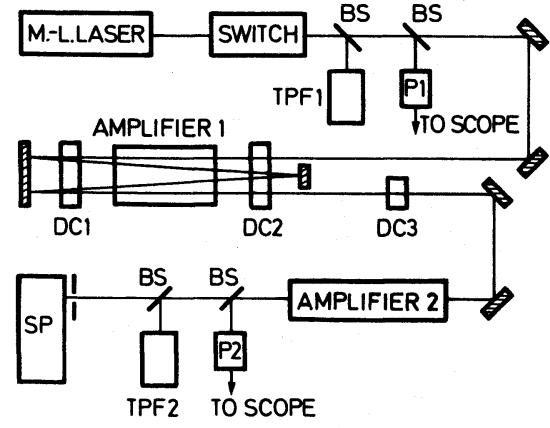

FIG. 4. Experimental setup for multiple transits through the absorber-amplifier system. Beam splitter BS; saturable absorber cells DC1 $\left(T_{0}=1.4 \times 10^{-2}\right)$, DC2 $\left(T_{0}=1.4 \times 10^{-2}\right)$, DC3 $\left(T_{0}=7 \times 10^{-3}\right)$; photodetectors P1, P2; two-photonfluorescence systems TPF1, TPF2; spectrometer SP.

the dye transmission, in the transient case the reduction of pulse duration is mainly an energy effect; the leading part of the pulse is strongly absorbed until the dye is sufficiently excited.

The results presented so far suggest the investigation of a system where a pulse is repeatedly shortened in a saturable absorber and subsequently amplified in a laser amplifier. We have made extensive calculations of multiple-absorber-amplifier system ${ }^{11}$ in the psec domain with widely varying parameters. In Fig. 3 a typical example is presented which indicates a situation of practical interest; the time dependence of the incident pulse (broken line) and of five subsequent pulses (solid lines) is shown for a system consisting of five absorbers with $T_{0}=2 \times 10^{-4}$ each, and four amplifiers each of optical gain $\gamma=4$. 1. An initial pulse duration of $\Delta t_{\mathrm{in}}=8 \mathrm{psec}$ and a peak intensity of the incident pulse of $I_{0}=1.1 \times 10^{9} \mathrm{~W} / \mathrm{cm}^{2}$ were assumed. In our calculations the individual pulse shape, after passing one absorber cell, was used as the initial condition for the passage through the following absorber. Amplification was considered to be intensity independent. Figure 3 gives a vivid picture of the drastic change in pulse shape after each absorber. Starting with $\Delta t_{1 \mathrm{n}}=8 \mathrm{psec}$, one calculates a final pulse of $\Delta t=0.65$ psec, i. e., a reduction factor of 12 . In this case the total transmission of the system is $3.2 \times 10^{-19}$. On the other hand, if a pulse of the same initial duration traverses just once a saturable absorber of the same transmission of $T_{0}$ $=3.2 \times 10^{-19}$, a most favorable reduction of the pulse duration is calculated to be a factor of 4.5 only (see Fig. 2). The absorber-amplifier system is more effective in pulse shortening because the distributed amplifiers bring the pulse intensity back to the optimum values for pulse shortening in the following absorbers. Our calculations show that the peak power of the final output pulse depends strongly on the optical gain $\gamma$. For values of $\gamma<4.1$ (input pulse and dye parameters as in Fig. 3), the succeeding pulses decrease quite strongly in peak intensity; they are of no practical interest. For gain values $\gamma>4.1$, the peak intensities of the pulses increase continually. For example, with the same parameters used in Fig. 3 but with $\gamma=4$. 3 , one calculates a final peak intensity of 30 times the incident peak intensity $I_{0}$; at the same time the pulse duration is reduced from $\Delta t_{\mathrm{in}}=8 \mathrm{psec}$ to $\Delta t=0.96 \mathrm{psec}$.

Experimentally, we investigated a folded absorber-amplifier system with parameters similar to the example discussed in Fig. 3. Our experimental setup is depicted schematically in Fig. 4. The optical switch provides us with a single pulse cut from the leading part of the pulse train. This pulse is analyzed by TPF 1 to be of $\Delta t_{\mathrm{in}} \sim 8 \mathrm{psec}$. The pulse passes effectively five times through absorbers and four times through an amplifying $\mathrm{Nd}$ : glass rod (amplifier 1). A final laser amplifier (amplifier 2) is used to increase the pulse for ready determination of the energy (P2), the pulse duration (TPF2), and the frequency bandwidth (spectrometer SP). The total transmission of the system was $T_{0}=10^{-17}$, and the total gain (amplifier 1) was approximately 300 . Our experimental results gave clear evidence of the drastic pulse shortening. The two-photon-fluorescence track (TPF2) showed a single sharp peak. We estimate a duration of the output pulse of $\Delta t=0.7 \mathrm{psec}$. With somewhat shorter input pulses we occasionally obtained output pulses of $\Delta t \leq 0.5 \mathrm{psec}$. The measured spectrum of the output pulse gave a frequency width of $\Delta \nu=9 \times 10^{11} \mathrm{~Hz}$ $\left(\Delta \tilde{\nu}=30 \mathrm{~cm}^{-1}\right)$. With these numbers we obtain a product $\Delta t \Delta \nu \simeq 0.6$, indicating that our pulses are nearly bandwidth limited. We have noticed in our investigations that the output pulse reacts strongly to changes of the input peak intensity $I_{0}$ and of the gain $\gamma .{ }^{12}$ This observation was expected from our calculations as discussed above in connection with Fig. 3.

The question now arises as to the shortest pulses which can be generated in an absorber-amplifier system of the type investigated here. While we feel that further pulse shortening is possible with more transits, there are several practical and principal limitations. With an increasing number of absorbers and amplifiers, the system becomes more sensitive to variations of $I_{0}$ and $\gamma$. As a result, the output pulse varies between small values of peak intensity and values of peak intensity high enough that other nonlinear effects occur in the dye solution or in the amplifier rod (e.g. , stimulated light scattering, self-phase-modulation). It should be noted that peak intensities exceeding $10^{10} \mathrm{~W} / \mathrm{cm}^{2}$ are readily generated in absorber-amplifier systems. A principal limit to the pulse duration is set by the spectral width of the amplifying medium; in Nd : glass the limiting pulse duration is expected to be $10^{-13} \mathrm{sec}$. In addition, for pulse durations shorter than the transverse relaxation time of the amplifer medium $\left[T_{2}(\mathrm{~A}) \simeq 0.6 \mathrm{psec}^{13}\right]$, coherent effects determined by the area under the electric field $\theta=(\mu / \hbar) \int_{-\infty}^{\infty} E d t$ have to be considered. In our case, with $I_{0}=10^{10} \mathrm{~W} / \mathrm{cm}^{2}, \Delta t=0.5 \mathrm{psec}$ and a dipole matrix element $\mu \simeq 10^{-19}$ esu, ${ }^{13,14}$ we estimate $\theta \simeq 0$. 5 ; under these conditions the amplifier gain is expected to be constant without breakup of the light pulse. ${ }^{14,15}$ The transverse relaxation time of the saturable absorber $T_{2}$ (dye) is estimated to be $T_{2}$ (dye) $\lesssim 10^{-13}$ sec. ${ }^{16,17}$ For $\Delta t \lesssim T_{2}$ (dye), the rate equations used in our calculations are not applicable any longer.

In conclusion, we wish to say that with properly adjusted absorbers and amplifiers, pulses of $\sim 5 \times 10^{-13} \mathrm{sec}$ have 
been obtained with the possibility of generating pulses of approximately $10^{-13} \mathrm{sec}$. The technique discussed here should be useful for pulse shortening in other laser systems as well.

${ }^{1}$ A. J. DeMaria, W. H. Glenn, Jr., M. J. Brienza, and M.E. Mack, Proc. IEEE 57, 2 (1969).

${ }^{2} \mathrm{D}$. von der Linde, IEEE J. Quantum Electron. (to be published).

${ }^{3}$ S. L. Shapiro and M.A. Duguay, Phys. Letters 28A, 698 (1969).

${ }^{4}$ D.J. Bradley, G. M.C. New, and S.J. Caughey, Phys. Letters 30A, 78 (1969).

${ }^{5}$ E. B. Treacy, Phys. Letters 28A, 34 (1968).

${ }^{6}$ M. J. Colles, Appl. Phys. Letters 19, 23 (1971).

${ }^{7}$ The material parameters of the dye used are $\tau=9.1 \mathrm{psec}$ and absorption cross section $\sigma=1.84 \times 10^{-16} \mathrm{~cm}^{2}$; see Eastman Kodak dye No. A9860 data release.

${ }^{8}$ J.D. Macomber, J. Appl. Phys. 38, 3525 (1967).
${ }^{9}$ A. Penzkofer, D. von der Linde, and A. Laubereau, Opt. Commun. (to be published).

${ }^{10} \mathrm{~J}$. A. Giordmaine, P. M. Rentzepis, S. L. Shapiro, and K.W. Wecht, Appl. Phys. Letters 11, 216 (1967).

${ }^{11}$ With an absorber-amplifier system, nsec light pulses have been shortened from 10 to $0.5 \mathrm{nsec}$ by N.G. Basov, P.G. Kryukov, V.S. Letokhov, and Yu. A. Matveets [Sov. Phys. JETP 29, 830 (1969)]. The steady-state calculations of this reference are not applicable for psec pulses.

${ }^{12}$ We have observed a decreasing amplifier gain for high peak intensities of the traversing pulse. This result has a stabilizing effect on our system. See also N.G. Basov, I. Kertes, P.G. Kryukov, Yu. V. Matveets, Yu. V. Senatskii, and S. V. Chekalin [Sov. Phys. JETP 33, 289 (1971)].

${ }^{13}$ E. Snitzer, Appl. Opt. 5, 1487 (1966).

${ }^{14}$ F. A. Hopf and M. O. Scully, Phys. Rev. 179, 399 (1969).

${ }^{15}$ P. G. Kryukov and V.S. Letokhov, Sov. Phys. Usp. 12, 641 (1970).

${ }^{16}$ M. Kasha, Discussions Faraday Soc. 9, 14 (1950).

${ }^{17}$ M. Hercher, Appl. Opt. 6, 947 (1967). 